to make an easy and careful inspection of the parts. Through fear of a long incision, much confusion and damage may and has resulted, by a blind groping after the obstructed portion. This found, be guided by its factorage. If there are long bridles of peritoneal bands, then simple division may be all that is required; if a twist or intussusception, perhaps these are as easily remedied. What shall be done if necro. sis of the intestinal tube has already supervened? One of two devices only is left for selection. Artificial anus, i. e. stitching the ends of the canal into the wound, with the hope of some further operative procedure, or resection of the necrosed portion, with a very careful adjustment of the divided ends and mesenteric attachment. This must be done in such a manner as to bring the peritoneal surfaces in approximation, snd in this way the wounded edges and mucous membrane are all turned into the intestinal cavity. Silk may be used, but at times proves an irritant. Catgut, if old, may be reliable, but frequently is absorbed too early, and properly prepared animal ligatures are to be preferred.

We are all familiar, indebted especially therefor to the careful studies of Sir Spencer Wells, with the rapid exudative repair processes which take place under the opposed peritoneal surfaces. In one instance, where we resected seven inches of the necrosed small intestine and death supervened thirty hours afterward, the exuded lymph had entirely encircled and covered in the approximated parts.

Having restored the continuity of the intestinal canal, readjust its relationships and close the abdominal wound. This is effected precisely as in ovariotomy, its essential factor being, as with the intestine, a careful approximation of the peritoneal surfaces. For this purpose let the stitches always be taken from within outward, inserting the needle about one-half an inch beyond the divided peritoneum. We believe gastrotomy should never be undertaken without the most careful antiseptic precautions. Many superficial students and opponents of antiseptic surgery refer to the spray as the summum bonum of the method, when, at best, in reality it is a minor factor. He who becomes familiar with the underlying broad principles of sound philosophic reasoning, the careful details of method, and the astounding array of demonstrated facts, will need little urging to give antiseptic surgery his enthusiastic support. Let it be held in remembrance that the whole endeavor of antiseptic surgery is not a wound benefited by carbolic acid or other medication, but thereby to secure a wound free from all germ infection, surgically clean, aseptic in character, as nearly as possible one like in condition to a subcutaneous injury.

If this has been effected, and this is not alone the theoretic aim of Mr. Lister and his followers, but is as a rule a sure accomplishment, then we may rest assured that our patient, if operated upon early, will probably recover. Great care in such operations must be taken not to reduce the temperature of the body, for heat is rapidly lost from the exposure of large surfaces of the peritoneum, and by the retention of its equilibrium a better capillary circulation is maintained, and shock is in large measure avoided.
Hæmorrhage is an exceptional and unimportant complication in this class of operations, since no large vessels are implicated. General and dangerous peritonitis is almost invariably septic, and with the exclusion of this factor, which may, as we have already claimed, be secured, a favorable prognosis can be given. In conclusion, let us be permitted to state that the operative surgeon should be guided by a wise consideration, based upon a thorough knowledge of the whole question, and that he is subject to the gravest of all responsibilities, remembering at the same time that the sins of omission are equally to be judged with those of commission.

\section{ON A NEW METHOD OF OBTAINING PURE PANCRE- ATIC JUICE.}

BY I.. B. TUCKERMAN, M.D.

[Read to the Section on Practice of Medicine, Materia Medica and Physiology, of Am. Med. Association, June, 1883.]

Physiologists are wont to employ one of two methods in obtaining pancreatic juice for purposes of experiment, viz., the method of Claude Bernard, and that of Ludwig and Bernstein. It is needless here to go into the details of either operation.

The method of Claude Bernard is open to the following objections:

I. Fixing, as it does, a temporary canula in the duct and collecting the secretion while the animal is under the shock of the operation, we can hardly assume the secretion to be normal.

2. This method offers but little opportunity for investigating variations in the character of the secretion dependent upon the period of digestion or upon the animal's diet.

The method of Ludwig and Bernstein is also open to objections, for :

I. The secretion of the fistulous tract kept open by the lead wire mingles with the secretion of the gland.

2. 'The irritation of the loop of wire in the pancreatic duct must must tend to keep the gland itself in a state of chronic irritation, thus altering the character of the secretion.

3. A considerable part of the pancreatic secretion is permanently diverted from the intestinal canal. This must more or less interfere with normal intestinal digestion and thus keep the animal in a pathological condition, thereby vitiating every secretion.

In view of these objections we think the conclusion of Dr. Flint a sound one-viz., "We are not disposed to admit that the fluid, collected by recent German observers from permanent fistulæ, represents physiological conditions." (Text Bk. of Phys. I 88 I, p. $27 \mathrm{I}$ ).

With a view of avoiding, as far as possible, the sources of error above entmmerated, we sought to devise a plan by which the pancreatic duct could, like Steno's duct, be catheterized at will. During the past few weeks we have succeeded in so doing by means of a permanent fistula, opening into that part of the duodenum which lies directly opposite the mouth of the pancreatic duct. The outer opening, and the outer flange of the canula used, are precisely 
like those of the ordinary canula for gastric fistula. The inner opening, however, is elliptical, its longer axis measuring $3 / 4$ inch, and its shorter $1 / 2$ inch. The inner flange is curved in the direction of the shorter axis of the opening, the radius of the curve being $3 / 8$ inch.

The details of Claude Bernard's method were followed as far as regards opening the abdominal cavity and drawing out the duodenum and head of the pancreas. The duodenum was then opened by a longitudinal incision at a point opposite the mouth of the duct, into which a probe was then passed. The canula was passed over the probe, the long axis of its inner opening being placed lengthwise of the gut. The latter was then stitched about the canula in such wise as to bring the mouth of the duct directly opposite the center of the inner opening of the canula. The position of the mouth of the duct was determined by means of a light thrown' down the canula from a head mirror. The parietal peritonæum was then stitched together and to the gut about the canula, and the wound in the abdomen closed.

When the animal had recovered, it was found that the mouth of the duct was still opposite the inner opening of the canula.

The accompanying figures will give an idea of the canula, and of its relations when in situ.

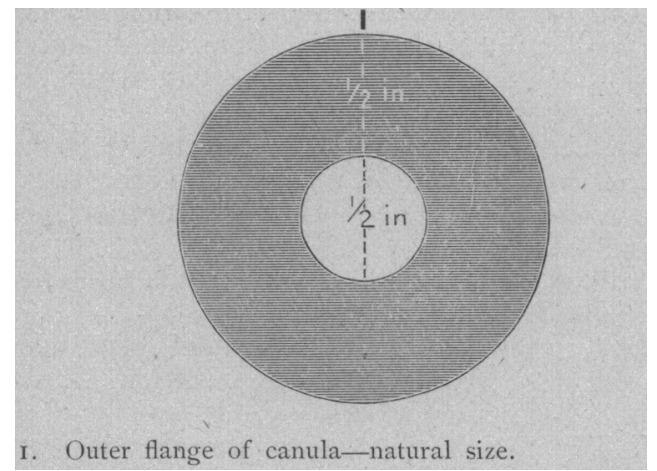

For catheterization of the cuct the animal is laid upon its left side, held still by assistants, and light is thrown down the canula by means of a head mirror. We have best succeeded with a small glass canula drawn down to about one-half inch in diameter and

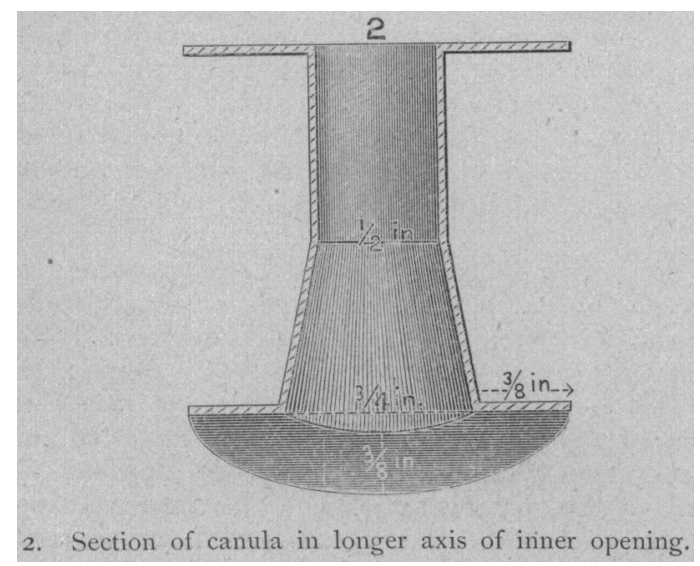

slightly bent about one-fourth inch from the small end.

The fluid thus obtained is perfectly transparent, distinctly alkaline and with marked amylolytic, pro-

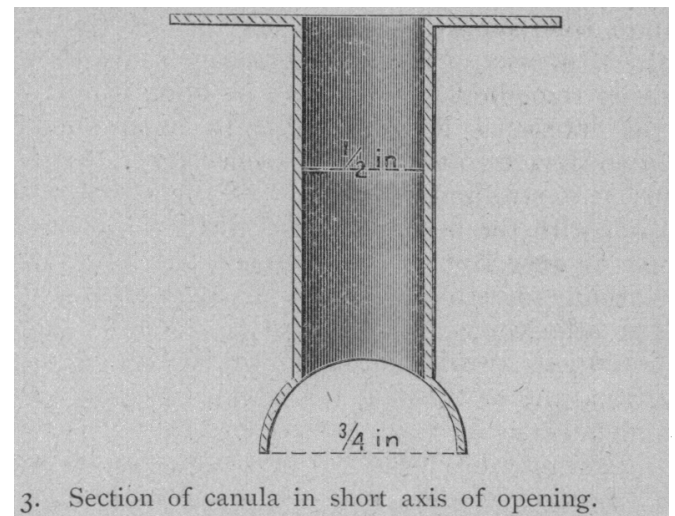

teolytic and emulsive properties. The advantages of this method are-

I. The animal is as nearly as possible in a physiological condition.

2. The fluid is obtained without causing the animal any marked inconvenience, and is unmixed with any other secretion.

3. The flund can be drawn at any time.

The difficulties are: I. No self-retaining catheter has as yet been devised, hence the experimenter must hold the small canula in place while the fluid is being drawn. This renders no little patience necessary in order to obtain the fluid in quantity.

2. Unless the mouth of the duct has been accurately fixed opposite the center of the inner opening of the permanent canula, the peristalsis of the gut

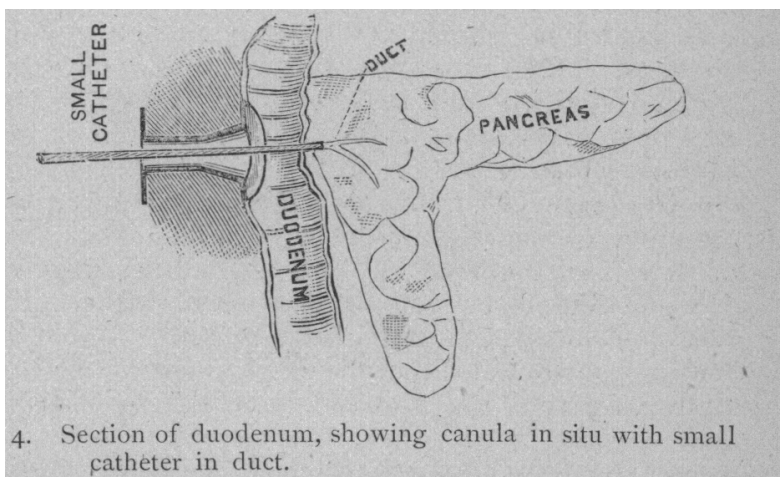

may at times draw the mouth of the duct out of sight. This makes it specially difficult to catheterize the duct while food is passing through the duodenum.

The lines of experiment to which this method would seem best adapted are :

I. To determine what, if any, are the variations in the character of the pancreatic secretion during different periods of digestion.

2. To discover whether difference in diet effects an appreciable difference in the relative amylolytic, proteolytic and emulsive properties of the pancreatic secretion. 
3. It may prove useful also, as Dr. H. P. Bowditch, of Boston, suggested to us, in studying the supposed relation of the spleen to the secretion of trypsin.

One other observation is perhaps worthy of mention here. It relates to the behavor of milk in the alimentary canal. 'Though physiologists tacitly assume that the primary digestion of milk is gastric, Roberis has shown ("On the Digestive Ferments," p. 4I) that "tryptic digestion of milk is rapir, and leaves only a slight residue, whereas peptic digestion is slow, and leaves a larger residue;" while Ewald ("Lectures on Digestion," p. 90) has proven that the pancreas, even in new born animals, is very active. Now, in this animal, milk, when fed alone, passes immediately and almost unchanged into the duodenum, where it appears mingled with bile and pancreatic secretion. If more extended observation shall show that the primary digestion of milk is wholly or in part tryptic rather than peptic, taking place in the duodenum rather than in the stomach, we have a physiological basis for a line of treatment clinically successful in certain digestive disorders of infancy, viz.: The exhibition of alkalies and drugs that, like ergot, nux vomica, etc., stimulate the unstriped muscular fiber. In such a case simple atony of the muscular walls of the stomach would seriously retard the process, even though the gastric secretion were perfectly normal. Moreover, one objection to the use of pancreatin, viz.: that it is destroyed in the stomach, is removed, if, when taken with milk or other fluids, it be rapidly passed into the duodenum.

\section{BLOOD-LETTING AS A REMEDYIN THE TREAT- MENT OF E ULAMPSIA PUERPERALIS AND "ACUTE PNEUMONIA."}

BY T. C. M'CUlloch, M.D., OIL CITY, PA.

On the 2 d day of July, 35 years ago, I was called to attend Mrs. John Carson, of Armstrong county, Pa., in labor with her third child.

She was a large, muscular woman about six feet in height and built in proportion. A short time before my arrival, she was delivered of a still born child. The womb was contracted, the afterbirth expelled. There was no hæmorrhage, and she was comfortably "put to bed." I say put to bed, as in those days women were nearly all delivered on the floor on their knees, and afterwards put to bed; apparently she was all right.

Whilst seated at the breakfast table enjoying my morning meal, after a seven mile ride, congratulating myself upon my "good luck," and easy made fee, I was startled by a scream from the nurse, followed by $\mathrm{d}$ sctor : doctor! On entering her room, I found my patientin a horrible convulsion. I say "horrible," for what can be more horrible to the practitioner than a woman convulsed, distorted and blackened by a puerperal convulsion; muscles convulsed, features distorted; respiration gives forth a hissing sound, froth issues from between the clenched teeth, the eyes are rolled upward, and spasmodically jerked from side to side. Truly the condition of the patient is "horrible" and well calculated to strike terror to the friends and alarm the doctor, especially if he is young and inexperienced.

At this period in medical therepeutics, there were but few remedies. Blood-letting, speedy delivery, and opiates in some form, were laid down as the esentials.

The room where the patient lay was small. The head and foot of the bed were wedged in between the wall and the partition, and as each proxysm occurred she actually shook the whole building. I bled this patient six times, once from both arms at the same time, allowing the blood to flow in full streams without regard to quantity. Her hair was cut off and cold applications applied to her head. She was cupped on the temples and back of the neck. She had twenty-three convulsions, occupying a period of twenty-four hours, during which time she was insensible. Her tongue was bitten, lips swollen, and face bloated, presenting an appearance unrecognizable to her friends.

Now, I think I hear the reader say, between the doctor and the disease she was a pretty well used up woman. But the convulsions ceased and she made a good recovery. She raised quite a large family. Had no return of the disease, but she was always bled once or twice prior to her confinements.

And let me say here that I believe I have prevented the occurrence of more cases of convulsions by timely blood-letting than I ever was called on to treat.

This lady afterwards gave birth to two sons who became prominent physicians - the oldest, Dr. Thomas Carson, who is at this time practicing in Saltsburg, Indiana County, $\mathrm{Pa}$; the other, the late Dr. John Carson, of Leechburg, Pa., who fell a martyr to his profession during a malignant epidemic of diphtheria, which prevailed in Armstrong county three winters ago. He died from a wound upon his hand, poisoned from the secretion while in the act of swabbing a child's throat.

This case of Mrs. Carson's I give as a typical case of the disease and the treatment thirty-five years ago.

Now, according to more recent views as to the cause, and the pathology of this disease, this was very bad treatment. Has the character of the disease changed? We know that the treatment has changed. But have there been any better results? Let us see. "I speak as to wise men, hear ye what I say." Upon looking over my Obstetrical Register, which I have kept faithfully from the first to the last case, noting all abnormal points in the labor, and writing out in full a history of all bad cases, I find I have seen fourteen cases of this disease, eight of which were patrons of my own; six I saw in consultation with other physicians. Of these eight cases seven were primpara. In one case I performed craniotomy; six were delivered by the forceps. But one, the case of Mrs. C., the child was born before the convulsions took place. All the cases were heroically bled, and all recovered but one.

My first case was in the summer of $\mathrm{I} 848$, my last case was in the fall 1878 -comprising a period of thirty years. 